\title{
Extended Formation Flight at Transonic Speeds
}

\author{
Andrew Ning \\ Brigham Young University - Provo, aning@byu.edu \\ Ilan Kroo \\ Stanford University \\ Michael Aftosmis \\ NASA Ames Research Center \\ Marian Nemec \\ NASA Ames Research Center \\ James Kless \\ NASA Ames Research Center
}

Follow this and additional works at: https://scholarsarchive.byu.edu/facpub

Part of the Mechanical Engineering Commons

\section{Original Publication Citation}

Ning, A., Kroo, I., Aftosmis, M. J., Nemec, M., and Kless, J. E., "Extended Formation Flight at Transonic Speeds," Journal of Aircraft, Vol. 51, No. 5, Sep. 2014, pp. 1501-1510. doi:10.2514/ $1 . \mathrm{C} 032385$

\section{BYU ScholarsArchive Citation}

Ning, Andrew; Kroo, Ilan; Aftosmis, Michael; Nemec, Marian; and Kless, James, "Extended Formation Flight at Transonic Speeds" (2014). Faculty Publications. 1674.

https://scholarsarchive.byu.edu/facpub/1674

This Peer-Reviewed Article is brought to you for free and open access by BYU ScholarsArchive. It has been accepted for inclusion in Faculty Publications by an authorized administrator of BYU ScholarsArchive. For more information, please contact ellen_amatangelo@byu.edu. 


\title{
Extended Formation Flight at Transonic Speeds
}

\author{
S. Andrew Ning* and Ilan Kroo ${ }^{\dagger}$ \\ Stanford University, Stanford, CA, 94305 \\ Michael J. Aftosmis, ${ }^{\ddagger}$ Marian Nemec, ${ }^{\S}$ and James E. Kless $₫$ \\ NASA Ames Research Center, Moffett Field, CA, 94035
}

\begin{abstract}
Aircraft flown in formation can realize significant reductions in induced drag by flying in regions of wake upwash. However, most transports fly at transonic speeds where the impact of compressibility on formation flight is not well understood. This study utilizes an Euler solver to analyze the inviscid aerodynamic forces and moments of transonic wing/body configurations flying in a two-aircraft formation. Formations with large streamwise separation distances (10-50 wingspans) are considered.

This work indicates that compressibility-related drag penalties in formation flight may be eliminated by slowing $2-3 \%$ below the nominal out-of-formation cruise Mach number (either at fixed lift coefficient or fixed altitude). The latter option has the additional benefit that the aerodynamic performance of the formation improves slightly at higher lift coefficients. While optimal in-formation lift coefficients are not as high as those estimated by incompressible analyses, modest increases in altitude can yield further improvements in aerodynamic efficiency. Increasing the lateral separation of the aircraft allows for slightly higher cruise speeds in exchange for higher induced drag. For the configurations examined here, a $1-2 \%$ reduction in Mach number combined with a lateral spacing increase of $5 \%$ span achieves a total formation drag savings of about $10 \%$.
\end{abstract}

\section{Nomenclature}

$\begin{array}{ll}A & \text { aspect ratio } \\ b_{0} & \text { separation distance between vortex pair } \\ c & \text { chord } \\ C_{D} & \text { drag coefficient } \\ C_{D p} & \text { parasitic drag coefficient } \\ C_{L} & \text { lift coefficient } \\ C_{p} & \text { pressure coefficient } \\ f & \text { functional } \\ L / D & \text { lift-to-drag ratio } \\ M_{\infty} & \text { freestream Mach number } \\ N_{c} & \text { number of cells } \\ r & \text { radial position } \\ S_{r e f} & \text { reference wing area } \\ V_{\theta} & \text { radial velocity } \\ x & \text { streamwise position }\end{array}$

*Ph.D., Department of Aeronautics and Astronautics, andrewning@alumni.stanford.edu, AIAA Member.

$\dagger$ Professor, Department of Aeronautics and Astronautics, AIAA Fellow.

¥Aerospace Engineer, Advanced Supercomputing Division, AIAA Associate Fellow.

$\S$ Senior Research Scientist, Science \& Technology Corp, AIAA Member.

`Aerospace Engineer, Science \& Technology Corp, AIAA Member. 
Symbols

$\Gamma_{0} \quad$ vortex circulation

$\lambda \quad$ taper ratio

$\Lambda_{Q C} \quad$ wing quarter-chord sweep

\section{Introduction}

With the world fleet projected to approximately double in size over the next twenty years, ${ }^{1,2}$ much effort is being devoted to further increase the efficiency of air transportation. Formation flight is one area of interest for its potential to significantly reduce fuel consumption of long range flights. Drag reduction through formation flight is not a new concept, and has been demonstrated in simulations, experiments, and flight tests. ${ }^{3-8}$

While the benefits of close formation flight have been known for some time, our recent analyses suggest that significant drag savings can be realized even when the aircraft are separated streamwise by five to fifty spans, in an arrangement we term "extended formation flight". ${ }^{9}$ These extended formations allow the aircraft to fly at safer separation distances while still retaining much of the formation benefits. However, for aircraft that fly at transonic speeds, some of the benefits may be negated or unrealizable due to compressibility effects. The upwash from a lead aircraft's wake can cause a large spanwise variation in induced angle of attack on the following aircraft (as high as approximately 6 degrees near the vortex core relative to far from the core). Transonic sections can have poor off-design performance at their design cruise speeds, and the modified load distribution may lead to shock formation, flow separation, buffet, and increased drag.

There are several ways to reduce the compressibility penalties due to formation flying including: alleviating the spanwise loading, redesigning the wing, flying further from the center of the vortex, and slowing down. In order to trim the aircraft in roll, some load redistribution is always necessary. Redesigning the wing to account for multiple design conditions may be beneficial, but presents its own challenges and would not help existing aircraft. Flying further from the center of the vortex will reduce the variation in induced angles of attack, but will also reduce the induced drag savings. Slowing down presents the most obvious solution, but longer flight times can negatively impact the economics of the aircraft's mission.

This study analyzes the inviscid aerodynamic performance of formations of aircraft flying at transonic speeds, separated streamwise by ten or more spans. These studies can be used to provide guidance for subsequent viscous solutions, studies using more detailed configurations, and experimental work. For simplicity, results focus on a two-aircraft formation of identical aircraft. Geometry changes (i.e., for trim) are not considered in the scope of this study.

The paper is divided into three main sections. First, the methodology is outlined. Second, a comparison between two different wake development methods is presented. Finally, inviscid performance for a two-aircraft formation with variation in Mach number, lateral position, and lift coefficient is evaluated.

\section{Methods}

The large streamwise separation distances involved in extended formation flight make high-fidelity simulation of the formation in a single domain computationally expensive. Fortunately, because of the large separation distances, the trailing aircraft has negligible influence on the lead aircraft. This allows the problem to be decomposed into three separable phases which are solved sequentially: lead aircraft, wake propagation, and trailing aircraft (Figure 1). First, the lift distribution of the lead aircraft evaluated is resolved its own domain. Second, this lift distribution is "rolled up" using a far-field analysis method augmented with empirical data. Finally, the propagated velocity field is imposed as an upstream boundary condition on a domain containing the trailing aircraft. Further details for each phase of the methodology are discussed below.

The solver used in this analysis is NASA's AERO package, a Cartesian mesh Euler solver with adjoint driven mesh adaptation. ${ }^{10-12}$ The mesh is adaptively constructed by refining cells that contribute most to discretization errors in user-selected functionals. The functional used in all results in this paper is $C_{L}^{2} /\left(\pi R C_{\text {Dinviscid }}\right)$. This functional is similar to span efficiency, but uses the net inviscid drag rather than the induced drag. Convergence in this functional allows for tight convergence in lift and drag simultaneously. NASA's AERO package is especially advantageous for this application because its mesh adaptation strategy allows for efficient propagation of the wake.

An alternative higher-fidelity methodology for the wake propagation in the first two phases was also 
explored. ${ }^{13}$ In the first phase, instead of extracting the lift distribution, the vorticity field on a plane one span behind the lead aircraft was resolved. This vorticity field was then used to initialize a 2D Navier-Stokes solver, which was propagated forward in time. The approach used in this paper is about three times faster than this high-fidelity approach, but more importantly results in a piecewise analytic model that can be easily evaluated at any level of grid fineness with minimal storage requirements. The previous study comparing the two methodologies found that the drag savings predicted by the two methods differed by less than a percent, and thus the higher-fidelity approach was deemed unnecessary for this analysis. For more detailed configurations, particularly those with deflected controls surfaces or for larger formations with multiple wake interaction, the higher-fidelity approach may be necessary.

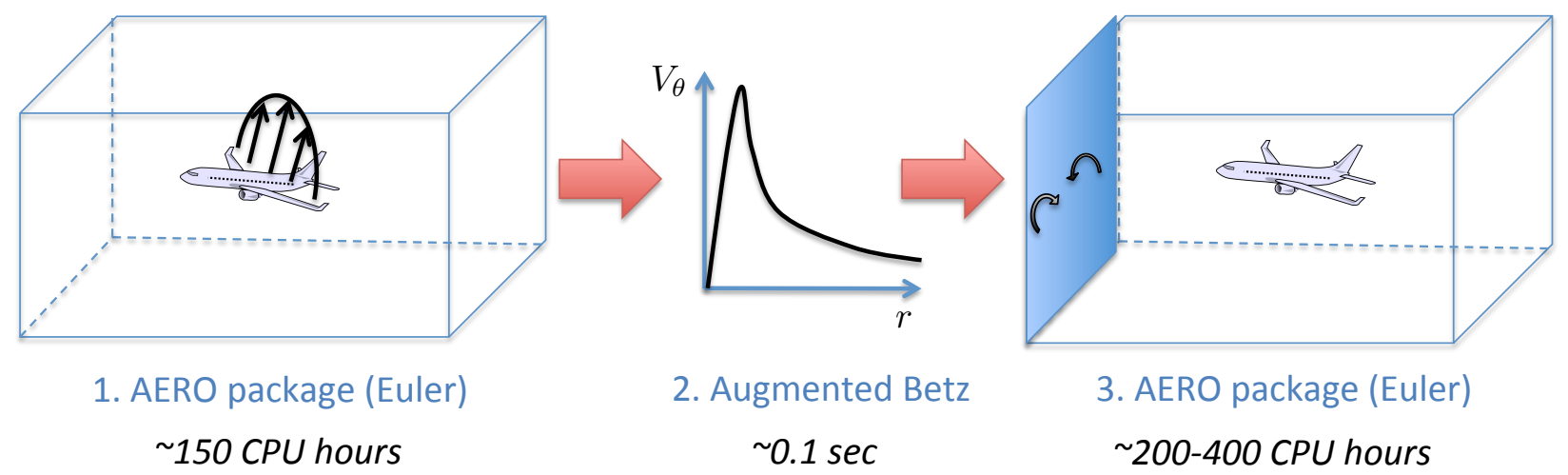

Figure 1. Overview of methodology. Lift distribution from lead aircraft is "rolled-up" using a far-field conservation of vorticity method. The resulting velocity field is then imposed as a boundary condition on a domain containing the trailing aircraft. The names of the various solvers, and some representative run-times are given for each stage (approximate timing for $1.6 \mathrm{GHz}$ Itanium 2 processors).

\section{A. Phase 1: Lift Distribution of Lead Wing}

The lift distribution of the lead aircraft could be estimated by a panel method or even be prescribed, but an Euler analysis provides a more accurate lift distribution and captures compressibility effects on the spanwise loading. This phase involved only a straightforward Euler analysis for a single aircraft. The domain boundaries were approximately 15 span lengths away from the aircraft in each direction, and a symmetry plane was used.

\section{B. Phase 2: Augmented Betz Wake Development}

As the wake is propagated downstream to distances of 10 to 50 spans, viscous effects can become important. However, the wake development process is still highly two-dimensional at these time scales, ${ }^{14}$ allowing for the use of a two-dimensional solver to a good approximation. The three-dimensional nature of the wake, as well as the effects of turbulence, would be important to consider if larger aircraft separation distances were desired.

In previous work, ${ }^{9}$ we developed a methodology to estimate the wake development process using Betz's method for computing a far-field vorticity distribution, ${ }^{15}$ experimental data on viscous core size, ${ }^{16}$ and a wake decay model based on the analytic/LES/experimental work of Holzäpfel. ${ }^{17}$ This method uses a functional form for the radial velocity as seen in Figure 2. It includes a linear solid-body rotation section, a quadratic section containing the peak velocity at the core, a section defined by the Betz methodology ${ }^{15}$ which is defined piecewise linearly with logarithmic spacing to better define the rapid velocity drop near the core, and the far field solution of a vortex with given circulation strength. The velocity at any point in the domain can then given be computed from the superposition of the induced velocities from all vortices defined on the upstream boundary (only 2 vortices for a 2-aircraft formation).

This approach has the advantage that it is very fast to evaluate, but it does lose some accuracy as the real vorticity distribution is not perfectly radially symmetric, nor does it follow this functional form exactly in a given direction. However, a perfectly accurate velocity field everywhere in the domain is not necessary 


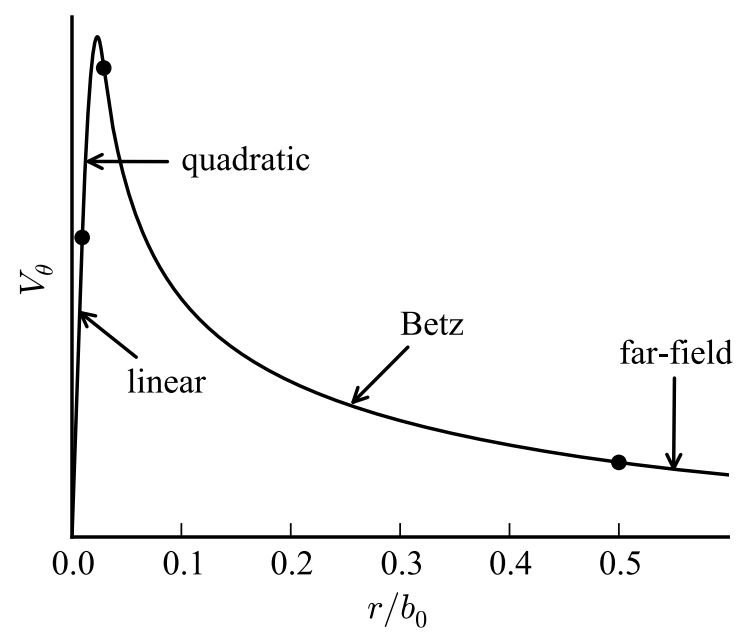

Figure 2. Functional form for the radial velocity from a vortex. There are four sections, a linear section, a quadratic section, a section defined by the Betz method, and a far-field vortex section.

as long as it is accurate in the region where the trailing aircraft is flying. Our previous studies found that this method was remarkably accurate as compared to a Navier-Stokes propagation method for this particular application. ${ }^{13}$

\section{Phase 3: Trailing Aircraft}

In the final phase, the velocity distribution from the lead aircraft's wake was imposed as an upstream boundary condition on a second domain containing the trailing aircraft. It was desirable to keep the upstream boundary close to the trailing aircraft in order to reduce computational costs, but still far enough away that the trailing aircraft had negligible impact on the wake development and that boundary effects were minimal. A boundary placement study suggested that a domain size of five spans in all directions was sufficient. Each cell volume was stretched streamwise with an aspect ratio of 1.7. This was done in order to reduce the number of cells needed to bring in the influence of the wake from the boundary. Convergence studies found this to be a good balance between the need for higher spanwise resolution in the wake region versus higher streamwise resolution near the body (both areas require high vertical resolution). An example of the evolution of the mesh for the trailing aircraft is seen in Figure 3 which shows a cut of the mesh in a plane just above the aircraft. In addition to the body refinement, the mesh was adaptively refined along the path of wake development to bring its influence in from the upstream boundary.

All cases for the trailing aircraft were run adaptively to approximately 25-30 million cells. This was a reasonable size given the number of cases and amount of resources available. However, at these cell counts, not all cases reached complete convergence. Richardson extrapolation was used to estimate the functional based on the results of the last three intermediate solutions from the mesh adaptation strategy. In the asymptotic convergence regime the functional can be related to the cell size as follows

$$
f=f^{*}+m\left(\frac{1}{N_{c}^{1 / 3}}\right)^{p}
$$

where $p$, the order of convergence, has been shown to be 2 for this code. ${ }^{10,12}$ This approach has been shown to work well with this CFD package in previous studies. ${ }^{18}$ A typical convergence plot and extrapolated line is shown in Figure 4.

The studies in this paper either prescribed a target lift coefficient and/or a position relative to the vortex center for the trailing aircraft. For the lead aircraft obtaining a target lift coefficient was straightforward, as the CFD solver automatically adjusted the freestream angle of attack in order to obtained a desired lift coefficient. This capability could not be used for the trailing aircraft domain, as the freestream angle of attack was required to remain at zero in order for the wake to propagate in the freestream direction. Instead, the 


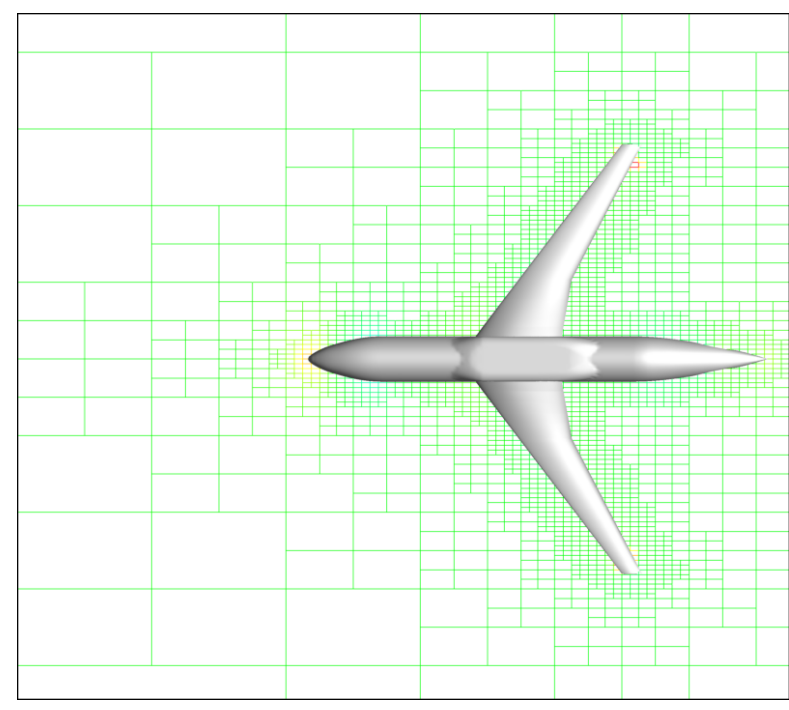

(a) initial mesh

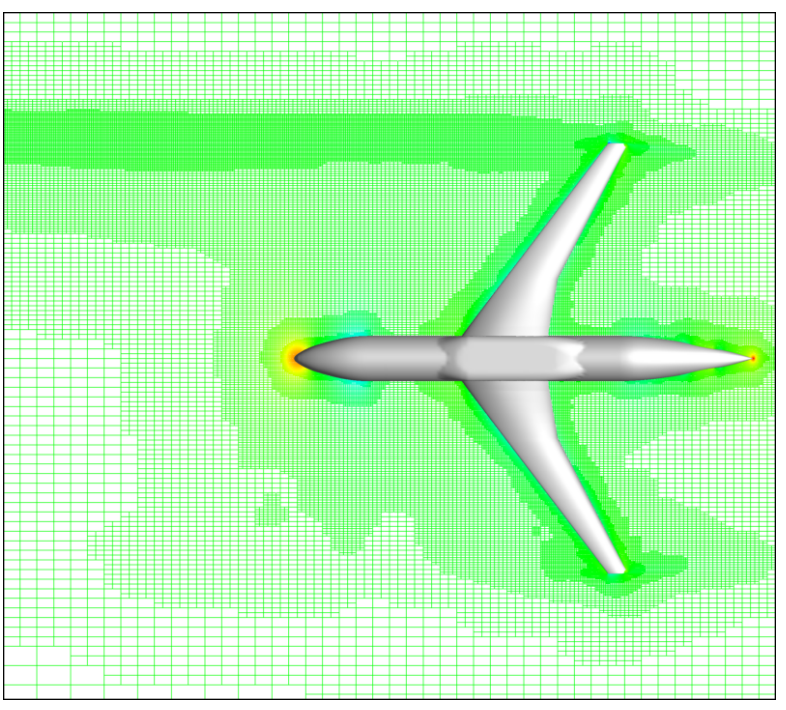

(b) final mesh

Figure 3. Cut plane passing near wingtip, with view looking up towards the bottom of the aircraft. Cells are colored by the local pressure. The mesh evolution is shown with the final mesh showing refinement of the vortex path from the upstream boundary.

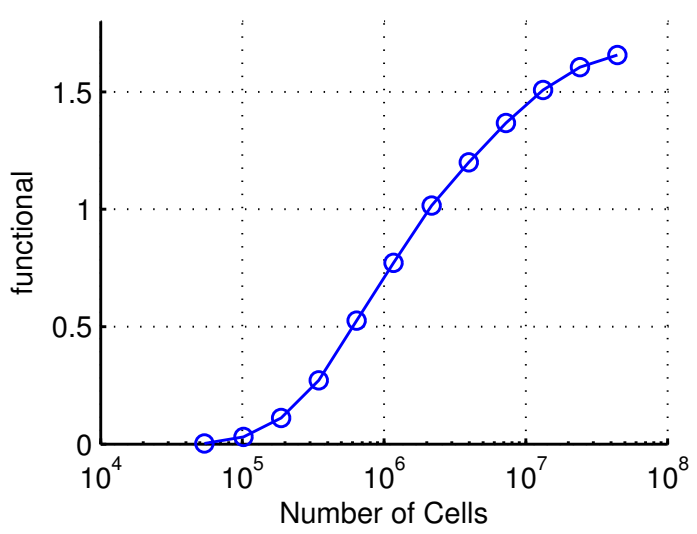

(a) Convergence in the functional $C_{L}^{2} /\left(\pi R C_{D \text { inviscid }}\right)$ with increasing cell count.

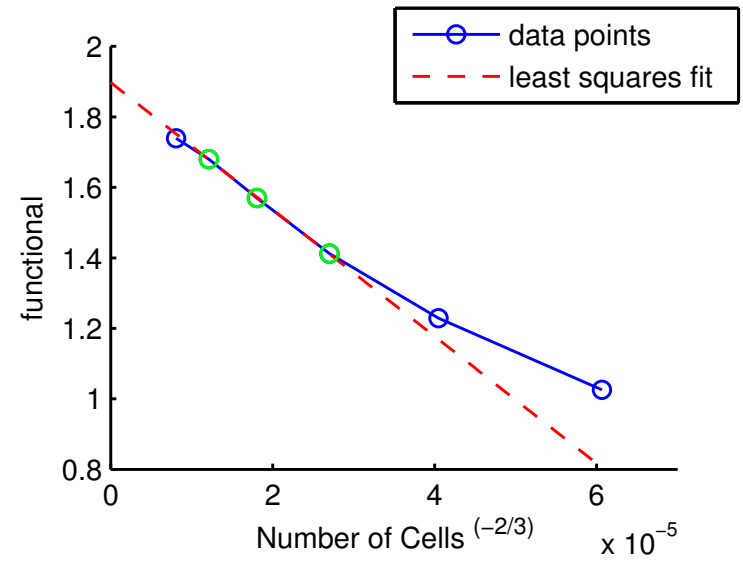

(b) Richardson extrapolation with least squares fit through green data points. Last point not included because most results terminate one iteration earlier.

Figure 4. A typical convergence plot for a transonic wing/body in-formation. This particular case was run out to $44 \mathrm{M}$ cells, though most results are terminated at $25 \mathrm{M}$ cells with continuum values estimated from Richardson extrapolation. 
geometry needed to be rotated to achieve the desired lift coefficient. However, the proper angle of attack to achieve a given lift coefficient in-formation was not known a priori. In order to avoid extra CFD iterations, a vortex lattice method combined with our simple wake model $^{9}$ was used to aid in the estimation of the proper angle of attack. While this low-fidelity method did not provide an accurate direct estimate of the angle of attack, it did provide a good estimate for the relative change in angle of attack required by moving in and out of formation. Since the appropriate out-of-formation angle of attack was already computed in the lead aircraft domain, a good estimate for the angle of attack required for the trailing aircraft could then be obtained. For all presented results, this method was sufficiently accurate to reach the desired lift coefficient within approximately $1 \%$ without the need to re-run the Euler solver a second time.

Similarly, for vortex positioning studies, in order to properly place the trailing aircraft in the domain it was necessary to estimate the vortex descent distance from the boundary of the domain to the wing of the trailing aircraft. The same simple wake model was used to estimate relative descent distances, and was also found to be accurate within $1 \%$ without iteration. A typical example, showing the location of the wingtip relative to the vortex is shown in Figure 5. This case targeted positioning the wingtip at the center of the vortex, and the actual position is within less than $1 \%$ of the aircraft's wingspan from the target position.

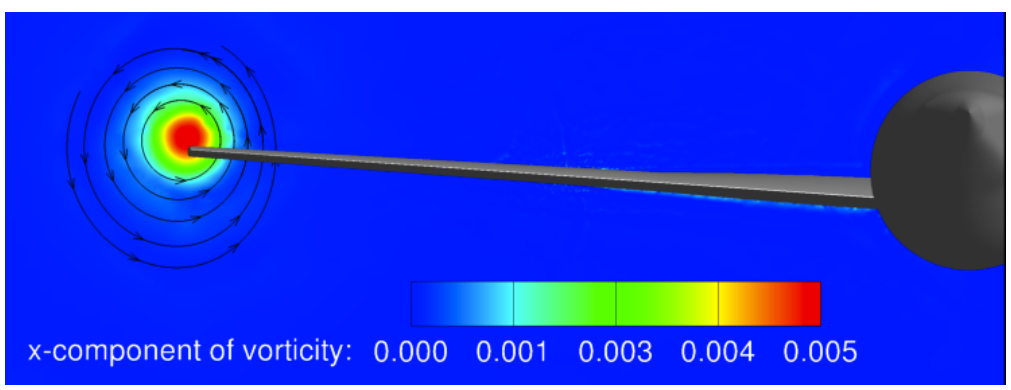

Figure 5. Contours of the component of vorticity in the freestream direction are shown on a cut through the wing for a transonic wing/body. A few confined streamlines near the wingtip are also shown.

\section{Two-Aircraft Formations}

The studies in this paper focus on formations of two identical transport aircraft separated streamwise by 20 wingspans. Variations in cruise Mach number, lateral positioning, and lift coefficient were examined.

\section{A. Geometries}

Two geometries were examined in this study. Both were transonic wing-body configurations with supercritical airfoils and blunt trailing edges. The first configuration was based on the DLR-F4 geometry used in the 1st AIAA CFD Drag Prediction Workshop. ${ }^{19}$ Inviscid solutions of that geometry have been shown to significantly over-predict lift, resulting in a negative angle of attack for the aircraft at the design lift coefficient of $0.5 .{ }^{20}$ In order to give a more realistic carry-through of lift across the fuselage, the wing was re-mounted on this geometry at a $4.5^{\circ}$ lower incidence angle so that the entire aircraft flew at $2^{\circ}$ incidence at the design conditions. In addition, the aft end of the fuselage was extended to a point in order to avoid issues with properly converging the base drag of the fuselage. This increased the fineness ratio of the fuselage by about $5.6 \%$.

The second geometry was the wing and body components of the Common Research Model used in the 4th AIAA CFD Drag Prediction Workshop. ${ }^{21}$ A summary of the design conditions for the two geometries is seen in Table 1, and isometric views of the aircraft are shown in Figure 6. All force coefficients are based on the same reference areas used in the above mentioned references namely, $S_{\text {ref }}=0.1454 \mathrm{~m}^{2}$ for Transport 1 and $S_{\text {ref }}=4,130 f t^{2}$ for Transport $2 .^{a}$

\footnotetext{
${ }^{a}$ No control surfaces were present on these geometries, thus all results were for untrimmed configurations. Subsequent work by the authors focuses on the effects of trim drag, and that work indicates that trimming can provide load alleviation on some of the more highly loaded sections, and slightly mitigate some of the compressibility penalties in the current work. ${ }^{22}$
} 
Table 1. Design Conditions of the Two Representative Geometries

\begin{tabular}{cccccc}
\hline \hline & $C_{L}$ & $M_{\infty}$ & $R$ & $\Lambda_{Q C}$ & $\lambda$ \\
\hline Transport 1 & 0.5 & $\sim 0.75$ & 9.4 & $25^{\circ}$ & 0.3 \\
Transport 2 & 0.5 & $\sim 0.85$ & 9.0 & $35^{\circ}$ & 0.275 \\
\hline \hline
\end{tabular}

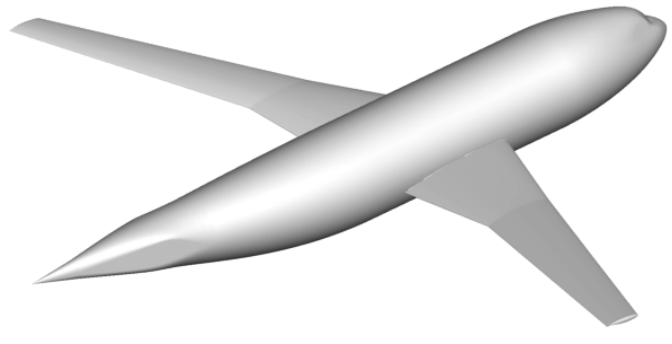

(a) Transport 1

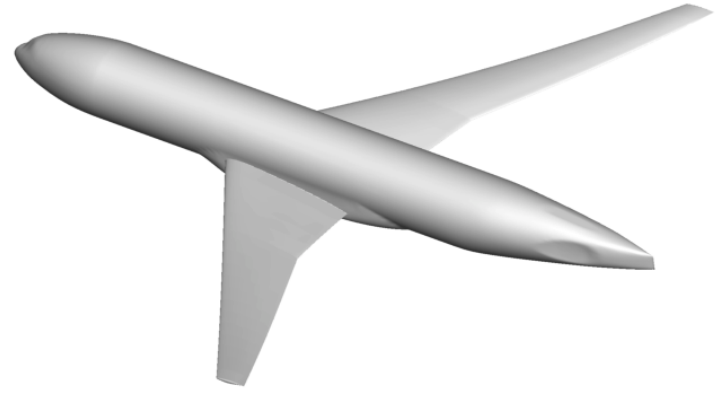

(b) Transport 2

Figure 6. Two transonic transport aircraft geometries. Both are wing/body configurations with supercritical sections.

\section{B. Variation in Mach Number}

To better understand the impact of formation flight at transonic speeds, an estimate for the out-offormation cruise speed for each aircraft was necessary. The drag divergence Mach number of an aircraft can be estimated by examining the variation in lift-to-drag ratio as a function of Mach number. Since the minimum cost speed for commercial aircraft tends to be somewhat faster than the maximum range speed, the cruise speed is usually faster than the speed that maximizes the lift-to-drag ratio. A simple method to estimate the drag divergence Mach number is the Mach number at which the lift-to-drag ratio drops by a couple percent from its maximum.

Since the Cartesian grid based simulation package solves the Euler equations, it provides only the inviscid component of the total drag, and thus an estimate of the viscous component of drag was needed. This was estimated simply by assuming that near the cruise speed, parasite drag accounted for $55 \%$ of the total drag. This is a fairly typical value for transport aircraft in cruise. ${ }^{23}$ A small variation in parasite drag with Mach number was also included based on the decrease in turbulence skin friction coefficient at higher Mach numbers. ${ }^{\mathrm{b}}$ This simple method was sufficient for our purposes because while the magnitude of the lift-to-drag ratio is sensitive to the particular choice of parameters, the relative variation in performance with Mach number is insensitive across a range of reasonable parameters. The lift-to-drag ratio for the two transports is shown in Figure 7. Based on the results of Figure 7 we took the nominal out-of-formation cruise Mach number to be $M_{\infty}=0.76$ for Transport 1 , and $M_{\infty}=0.83$ for Transport 2 .

Predicting the optimal in-formation Mach number is more complex. The inviscid drag for both the lead and trailing aircraft in a two-aircraft extended formation is shown in Figure 8 for the two different transports. All cases were run at a constant lift coefficient of 0.5 for both lead and follower aircraft, with the trailing aircraft positioned so that its wingtip was at the center of the incoming vortex. While both aircraft see a similar trend with increased drag at higher Mach numbers, the rate of drag increase was slightly higher for the trailing aircraft.

Because the trends in the drag rise curves are similar, it may appear that the in-formation cruise speed should be essentially the same as the out-of-formation cruise speed. However, because the calculations are inviscid, a closer examination of the pressure distribution on the wing is necessary. Formation-induced shocks may not always be large enough to manifest as large increases in compressibility drag, but could still be strong enough to separate the flow on the wing and make the aircraft unflyable due to buffet. As a

\footnotetext{
bhttp://adg.stanford.edu/aa241/drag/skinfriction.html, accessed June 13, 2011
} 


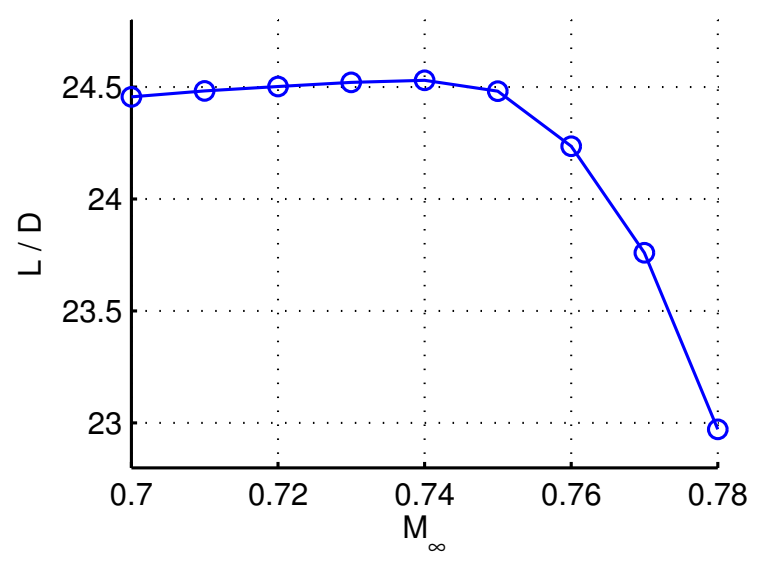

(a) Transport 1

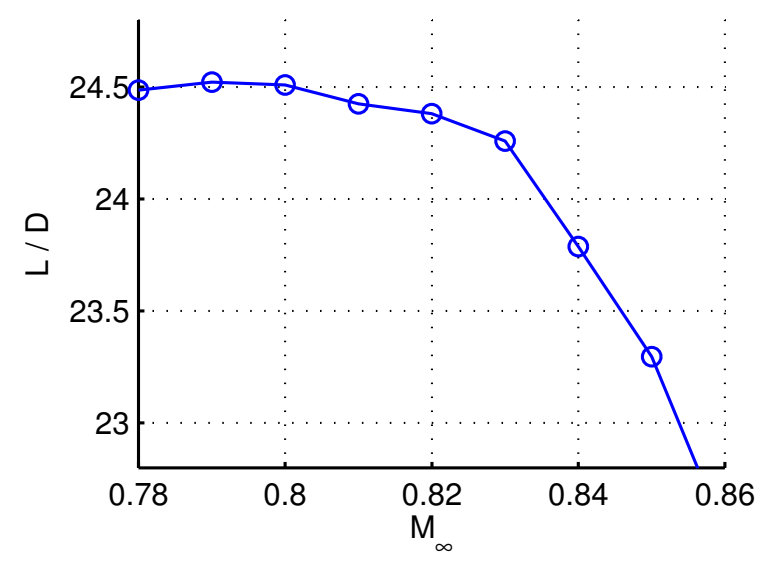

(b) Transport 2

Figure 7. Aircraft lift-to-drag ratio as a function of freestream Mach number (out-of-formation).

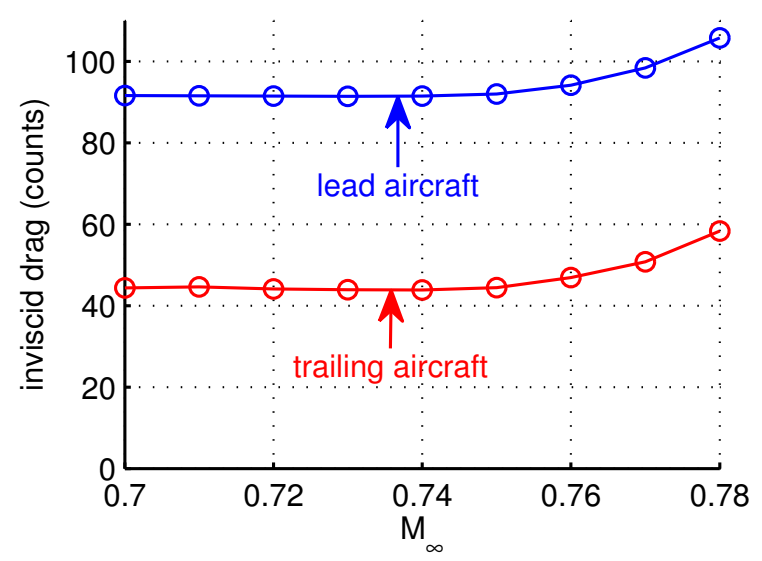

(a) Transport 1

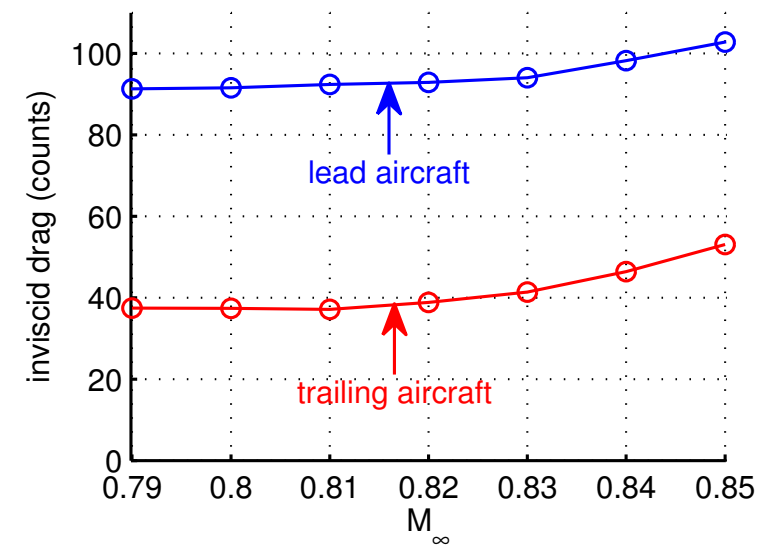

(b) Transport 2

Figure 8. Inviscid drag for lead and trailing aircraft as a function of Mach number for a two-aircraft homogeneous formation. Lift coefficient is held constant at $C_{L}=0.5$. For all in-formation cases the trailing aircraft's wing tip is positioned at the center of the incoming vortex. 
simple measure of shock strength, the maximum Mach number on the aft half of the local wing section was computed as a function of spanwise position for Transport 2 (Figure 9). The variation was computed at three different Mach numbers. Only the half of the wing nearest the incoming vortex is shown, as the other half of the wing is essentially unaffected by the vortex upwash. For reference, the out-of-formation cruise condition is also shown. For this analysis, any shocks stronger than the reference condition were deemed unacceptable. At the out-of-formation cruise speed, the trailing aircraft in formation experiences significantly stronger shocks near the incoming vortex. The figure suggests that slowing down at least $1.5 \%$ below the nominal out-of-formation cruise speed may be sufficient to alleviate the formation-induced compressibility penalties.

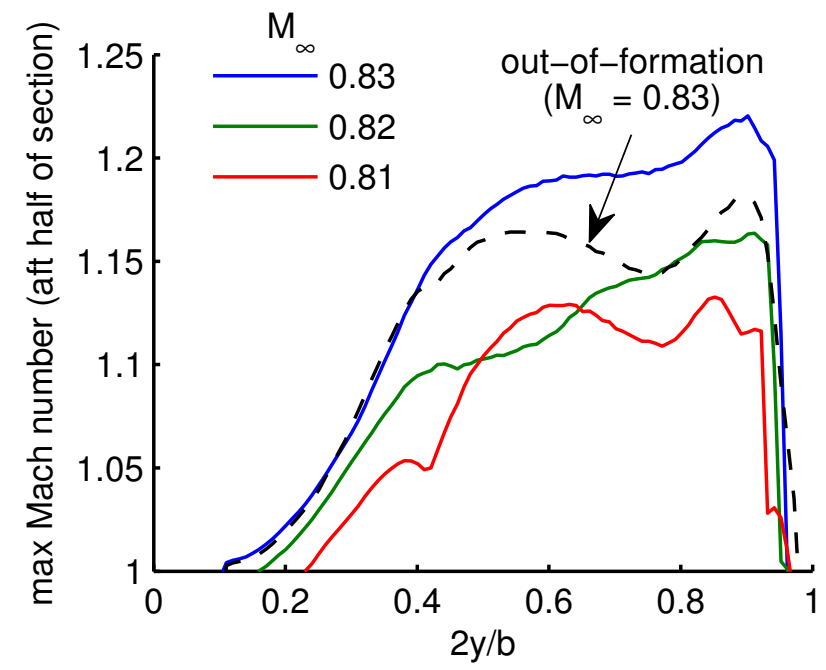

Figure 9. Maximum Mach number on the aft half of the local section as a function of spanwise position for Transport 2 (only half of wing nearest incoming vortex shown). In-formation cases are shown for three different Mach numbers, and the out-of-formation case in shown at the cruise condition.

While the simple measure of shock strength provides some insight into the impact of flying at transonic speeds in formation, the pressure distribution across the wing was also examined in more detail at the conditions of interest. The $C_{p}$ distribution on the upper surface of the wing for the half of the wing nearest the vortex is shown in Figure 10 for Transport 1 and Figure 11 for Transport 2. The wing is shown at the cruise Mach number out-of-formation, the cruise Mach number in-formation, and at a reduced Mach number in-formation. $C_{p}$ cuts are also shown at a few stations along the wing. The reduced Mach number in the figures is approximately $2.5 \%$ below the out-of-formation drag-divergence Mach number.

The results suggest that by slowing down about 1.5-2.5\% below the nominal out-of-formation cruise speed, the compressibility penalties of flying in-formation can be essentially eliminated. Shock strength is reduced to or below that of the wing at its out-for-formation cruise condition. The main difference in the formation cases is the stronger nose suction peak. This is generally acceptable, as the adverse pressure gradient on the back side of the peak should be tolerable near the nose of the airfoil where the boundary layer is still strong.

If the formation flies at a slower cruise speed, then the formation would also need to fly at a lower altitude or at a higher lift coefficient than the out-of-formation conditions (or some combination of both). A 2.5\% reduction in Mach number corresponds roughly to a 1,000 ft drop in cruise altitude at fixed lift coefficient, or a $5 \%$ increase in lift coefficient at fixed altitude. A higher lift coefficient may be desirable, as incompressible analyses suggest that the optimal lift coefficient in-formation is higher then the out-of-formation optimum. ${ }^{6}$ However, this result does not necessarily hold at transonic speeds and is explored further in Section D.

Finally, the Euler results are compared to the incompressible methodology used in our past work ${ }^{9}$ in Figure 12. The geometry used in the vortex-lattice model was re-twisted so that the out-of-formation lift distribution was reasonably well matched at the cruise condition. The figure shows the formation drag fraction, which is the total drag of all the aircraft in-formation relative to the drag of all aircraft out-offormation at the cruise condition. Viscous drag was estimated using the simple method described previously. In plotting the formation drag fraction it was assumed that the magnitude of the viscous component of drag did not change when the aircraft were in formation. From the figure we see that the low-fidelity results agree 

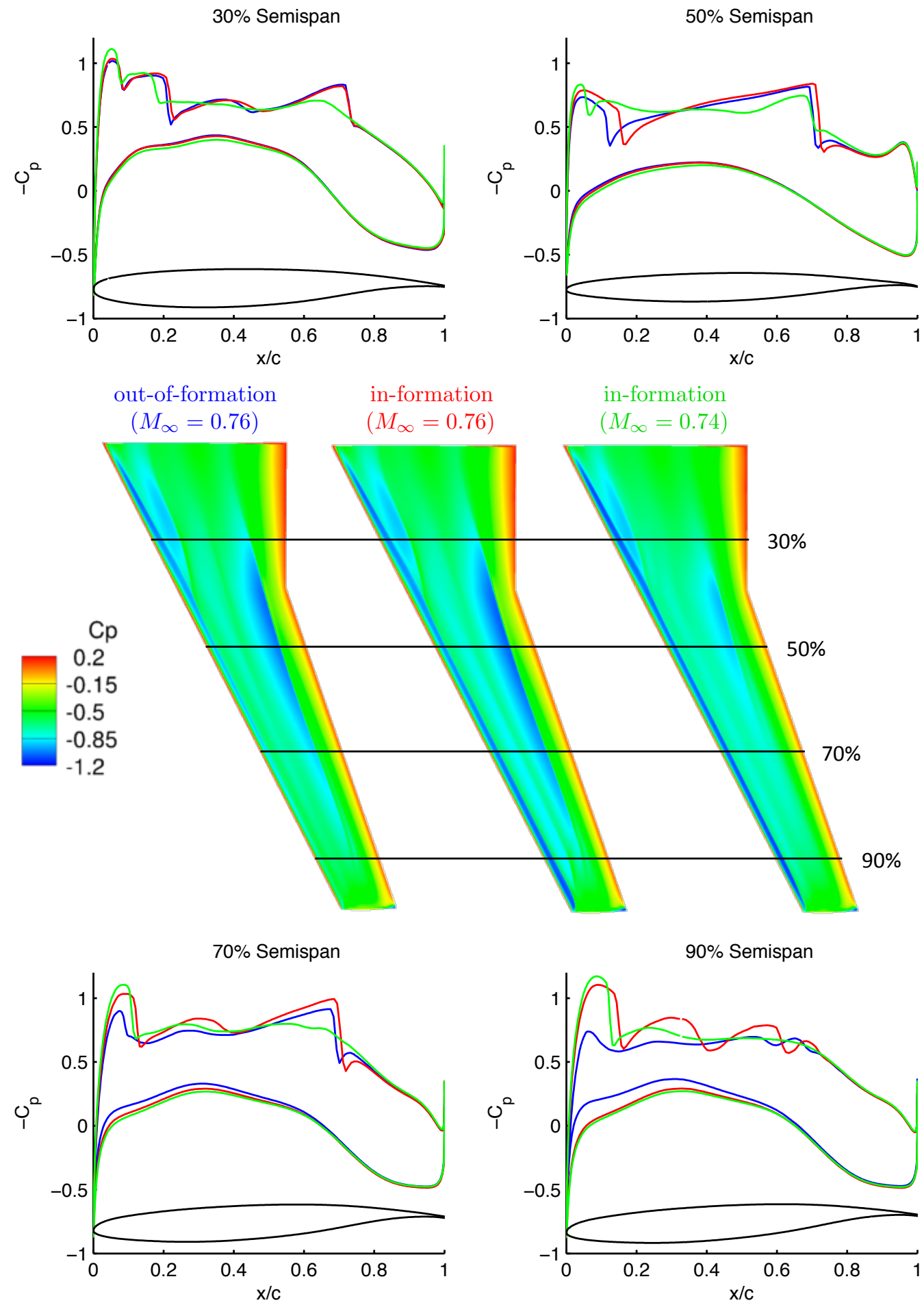

Figure 10. $C_{p}$ contours on upper surface of wing of Transport 1 at three conditions: out-of-formation at the cruise speed, in-formation at the cruise speed, and in-formation at a lower Mach number. For the in-formation cases, the aircraft is the trailing aircraft in a two-aircraft formation, and the half of the wing shown is the one closest to the incoming vortex. The $C_{p}$ distribution is also shown at select cuts through the wing. 

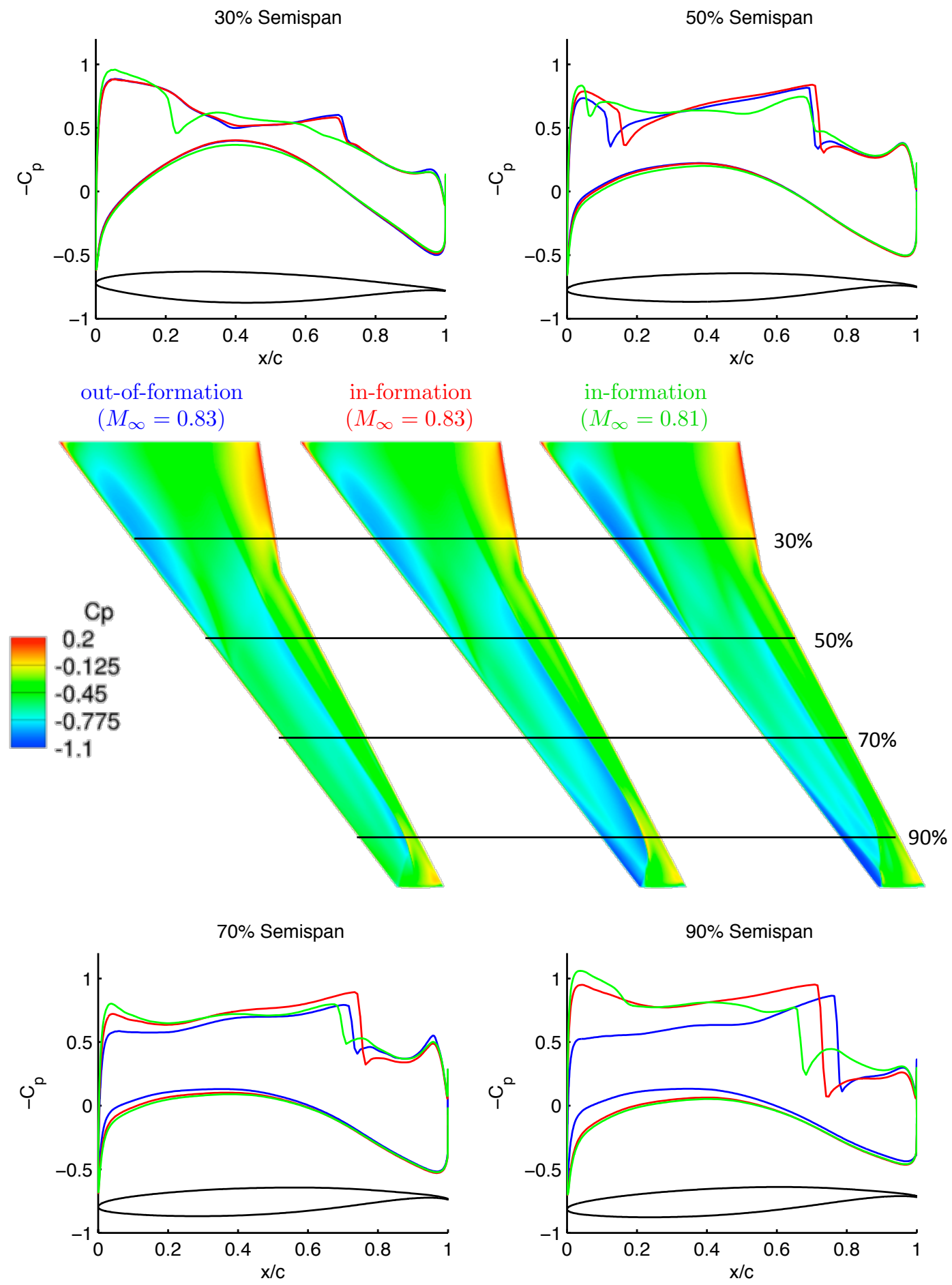

Figure 11. $C_{p}$ contours on upper surface of wing of Transport 2 at three conditions: out-of-formation at the cruise speed, in-formation at the cruise speed, and in-formation at a lower Mach number. For the in-formation cases, the aircraft is the trailing aircraft in a two-aircraft formation, and the half of the wing shown is the one closest to the incoming vortex. The $C_{p}$ distribution is also shown at select cuts through the wing. 
well with the Euler solutions until compressibility effects start to become significant.

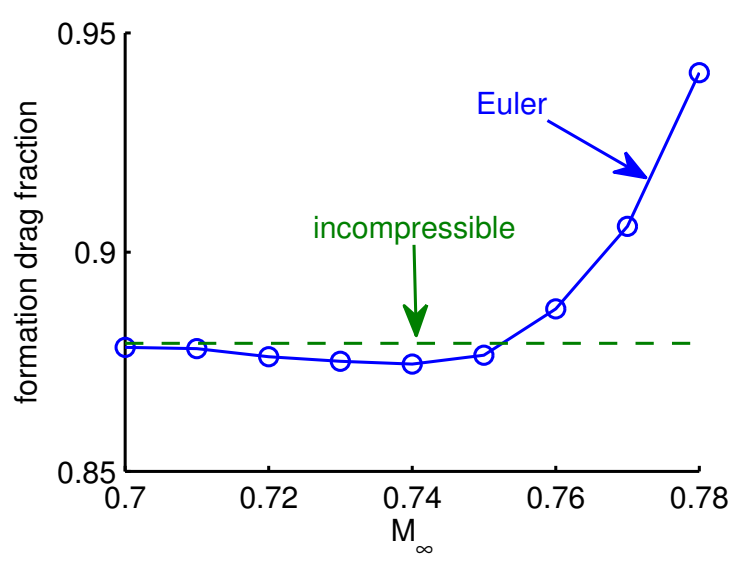

(a) Transport 1

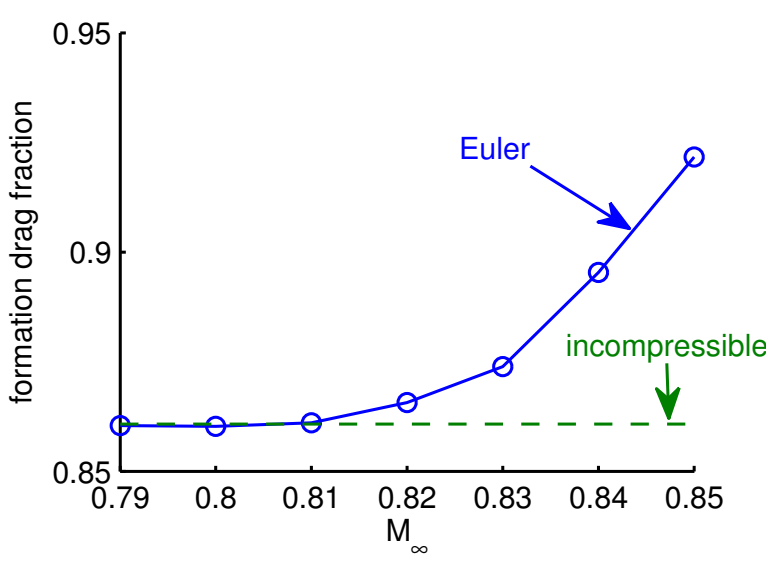

(b) Transport 2

Figure 12. Comparison of the formation drag fraction between an Euler solution and an incompressible aerodynamics analysis with far-field wake models.

\section{Variation in Lateral Positioning}

While slowing down can reduce the formation-induced compressibility penalties, there are economic incentives to flying faster. By flying further from the vortex center some of the compressibility penalties may be reduced without having to slow down as much. The following study examines the effect of varying the lateral separation, while keeping the vertical position aligned with the center of the vortex. Vertical separation is expected to have a similar effect, although the distances would differ as formation drag savings are more sensitive to vertical separation. ${ }^{9}$ The definition for the relative spacing is seen in Figure 13. The variation in formation drag fraction with lateral spacing is shown in Figure 14 for three different Mach numbers, beginning with the cruise Mach number. At all lateral positions, a small reduction in formation drag is realized by reducing the Mach number, however further decreases in Mach number exhibit quickly diminishing benefits. The figure also suggests that the incompressible analysis is quite accurate in capturing the trends reflected in the Euler solutions.

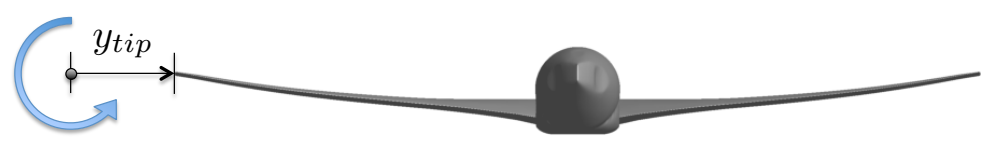

Figure 13. Positioning defined from center of nearest vortex from upstream aircraft to wingtip of trailing aircraft.

The compressibility penalty in-formation was examined using the metric of local Mach number on the aft half of the section in Figure 15 (Transport 2). At the out-of-formation cruise Mach number, none of the separation distances examined return the shock strength to the out-formation levels. The shock strength does become comparable when the trailing aircraft is about 0.1 spans or more from the vortex center, but at these larger separation distances the inviscid drag savings of the formation is reduced by about $10 \%$ or more. Most of the reduction in shock strength appears to occur for $y_{\text {tip }} / b \leq 0.05$. Larger spacings provide little additional benefit, especially considering the large loss in induced drag savings.

A closer examination of the pressure contours on the wing suggested a similar conclusion. A vortex septation distance of $y_{\text {tip }} / b=0.05$ was not sufficient to return shock strength to out-of-formation levels, but the same spacing combined with a 1.5\% reduction in Mach number eliminated the formation-induced compressibility penalties. Similar results were obtained for both transport geometries. These numbers are not meant to suggest optimal operating conditions; the tradeoffs in drag savings, and cruise speed need to 


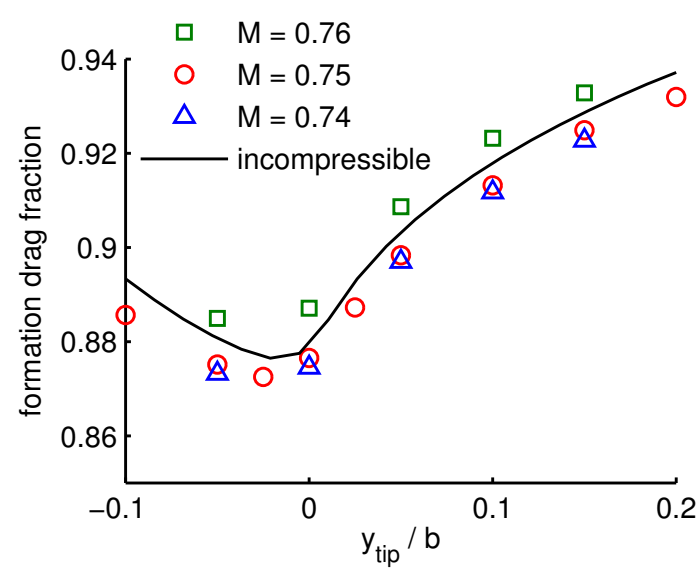

(a) Transport 1

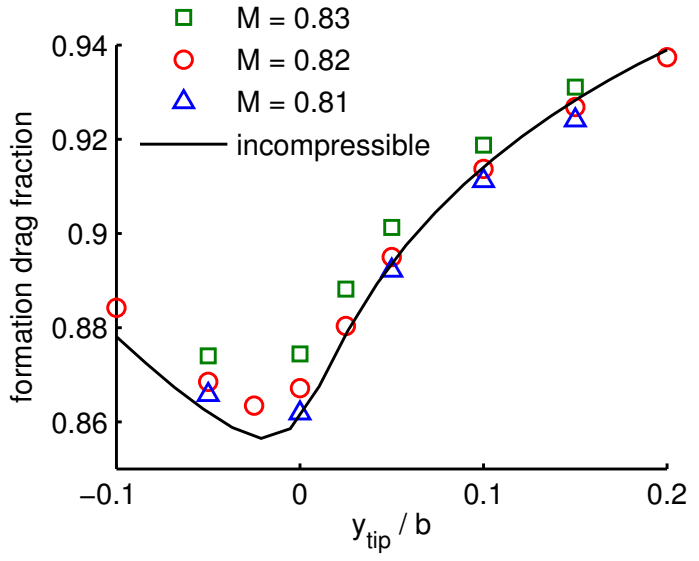

(b) Transport 2

Figure 14. Formation drag fraction of the formation as a function of the position of the trailing aircraft's wingtip relative to the incoming vortex center. Results are shown for three different Mach numbers, beginning with the cruise Mach number. Also shown for comparison is a low-fidelity incompressible estimate.

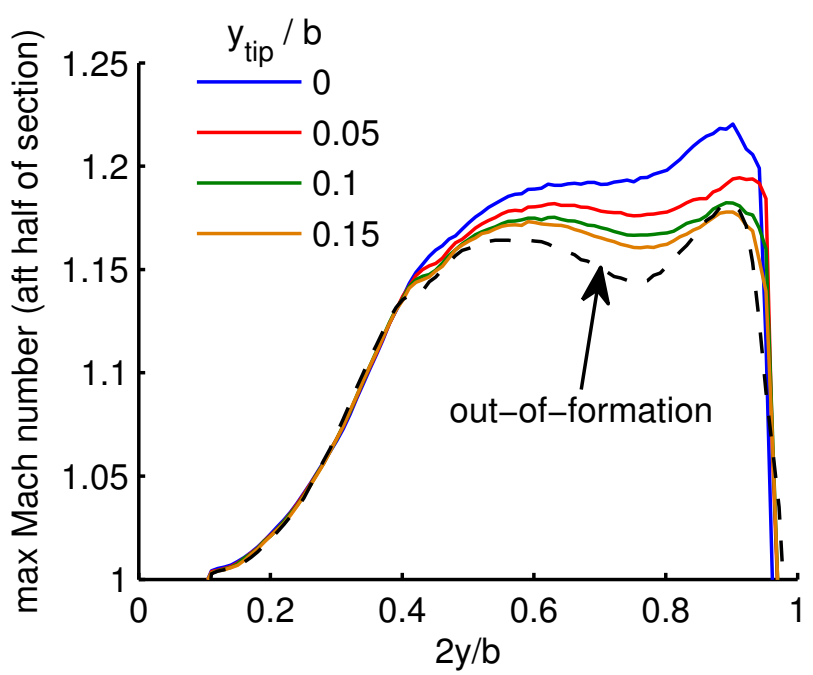

Figure 15. Maximum Mach number on aft half of the local section as a function of spanwise position for Transport 2 (only half of wing nearest incoming vortex shown). In-formation cases are shown for four different lateral spacings, and the out-of-formation condition is shown for reference. 
be determined in the context of a specific mission where the relative costs of fuel consumption and flight time can be assessed. One of our recent studies has examined these tradeoffs for optimized flight trajectories and schedules across an airline and alliance of airlines. ${ }^{24}$

\section{Variation in Lift Coefficient}

Incompressible analyses suggest that the optimal lift coefficient in formation is higher than the optimal lift coefficient flying solo. However, compressibility penalties will decrease the optimal lift coefficient when flying in formation at transonic speeds. Because this analysis depended on the tradeoff in induced versus all other forms of drag, an estimate for the viscous component of drag was needed. Parasite drag was estimated simply as discussed previously. Additionally, because the lift coefficient was changing, a small viscous increment was added as a function of lift coefficient:

$$
C_{D i \text { viscous }}=K C_{D p} C_{L}^{2}
$$

where $\mathrm{K}$ is based on flight tests of commercial transports. ${ }^{25}$ While the specific values of lift-to-drag ratio, and the optimal lift coefficient will vary with the value of the assumed parameters, the general conclusions of this section remain the same for reasonable variation in these parameters.

Figure 16 shows the variation in formation drag fraction as a function of lift coefficient for a few different Mach numbers, along with the incompressible estimate. As expected, increases in the freestream Mach number cause the optimal lift coefficient to decrease. Still, moderate increases in lift coefficient may be realizable, and can yield a small performance benefit. As already noted, flying slower is a useful approach to eliminating the compressibility penalties associated with flying in-formation at transonic speeds. By simultaneously flying at a higher lift coefficient, the formation can realize a small increase in performance while maintaining design altitude. Figure 17 compares the pressure distribution for Transport 2 at its out-of-formation cruise condition, and in-formation at a reduced Mach number but at fixed altitude.

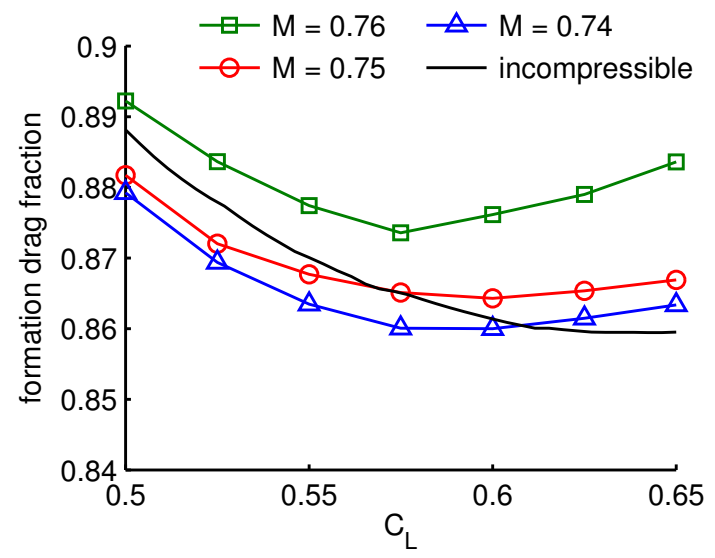

(a) Transport 1

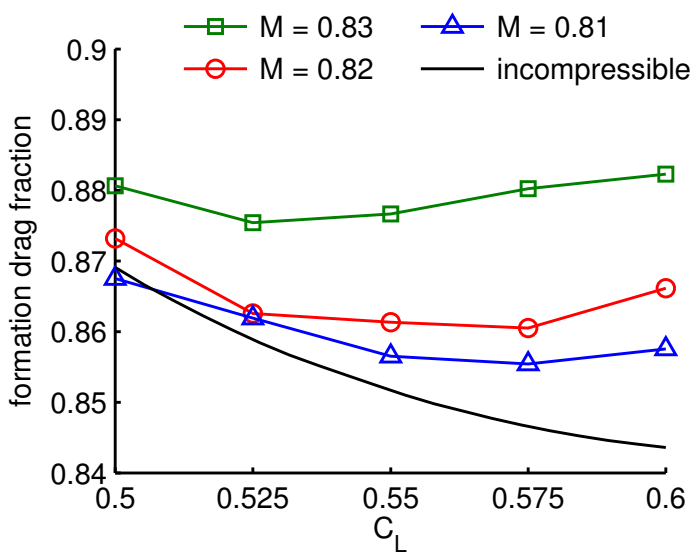

(b) Transport 2

Figure 16. Variation in formation drag fraction as a function of lift coefficient. As expected, formation flight has improved aerodynamic performance at higher lift coefficients, but not as high as predicted by incompressible analyses. 

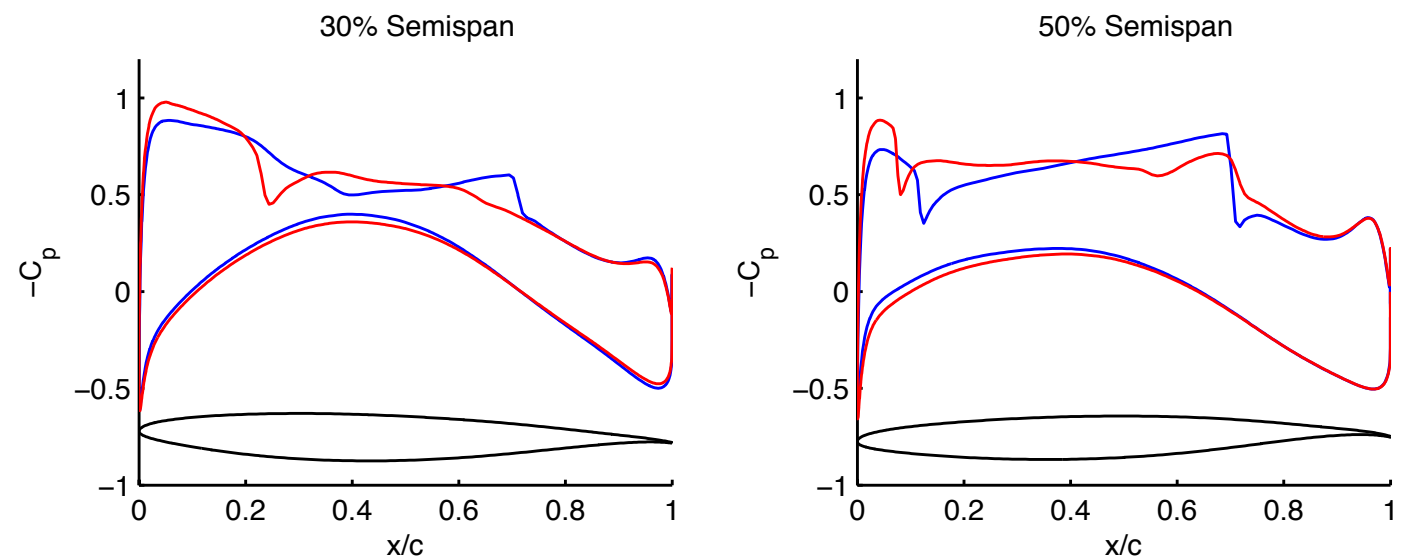

out-of-formation

in-formation

$\left(M_{\infty}=0.83, C_{L}=0.5\right) \quad\left(M_{\infty}=0.81, C_{L}=0.525\right)$

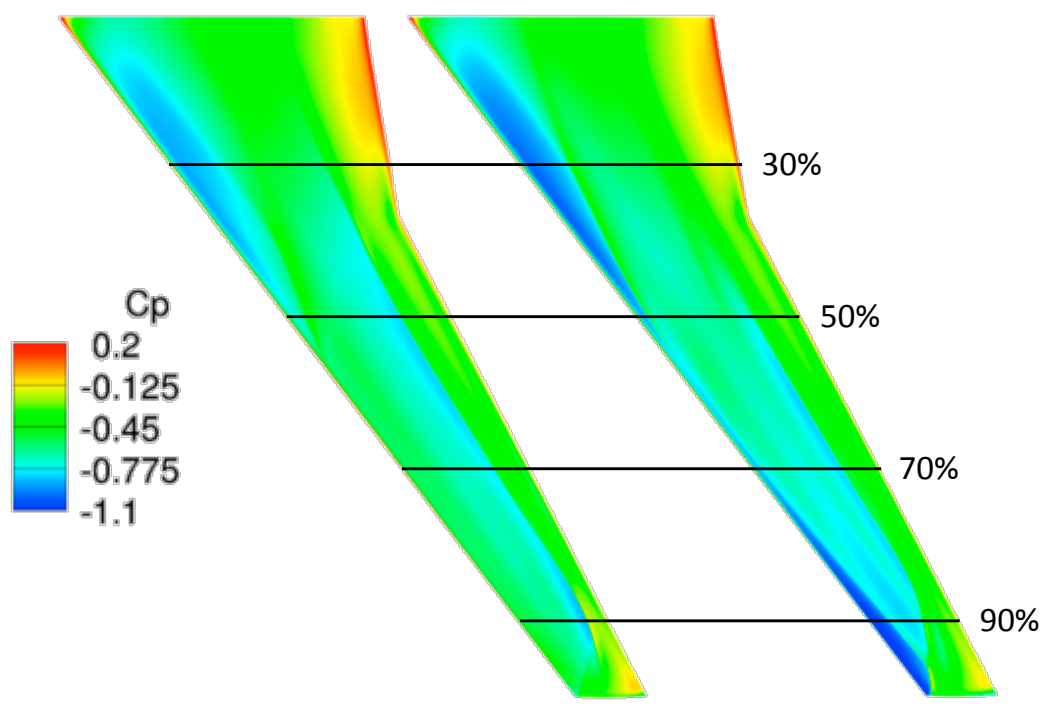

70\% Semispan

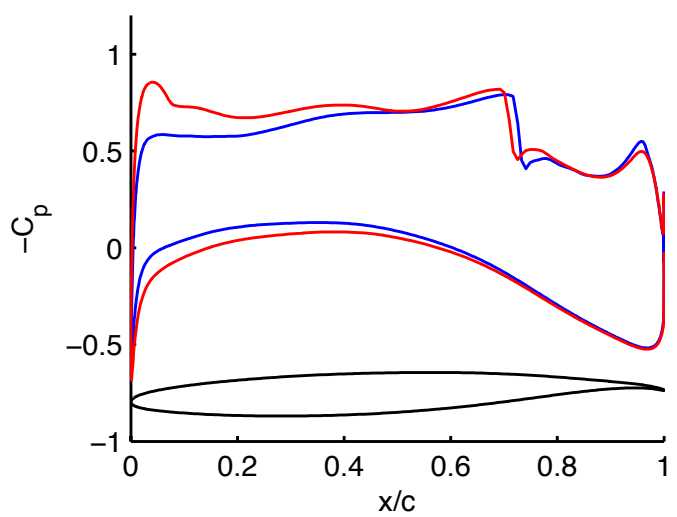

$90 \%$ Semispan

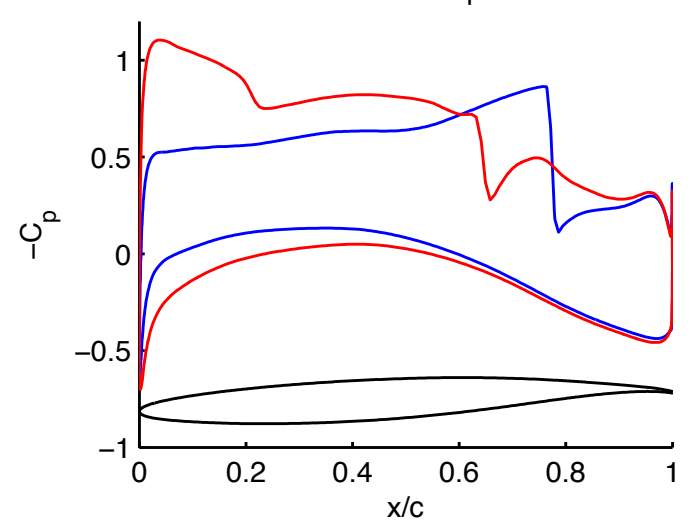

Figure 17. Comparison between Transport 2 at its out-of-formation cruise condition, and in a two-aircraft formation with a reduced Mach number but fixed altitude. 


\section{Conclusion}

Euler simulations at transonic speeds have shown that compressibility effects can rapidly erode the savings offered by formation flight at today's typical cruise speeds (near the drag divergence Mach number). Detailed simulation of homogeneous two-aircraft formations showed local formation-induced shock strengthening which contributes to premature drag rise and has a strong potential to lead to separation or buffet. However, slowing down by $2.5 \%$ in Mach number essentially eliminates the formation-induced compressibility penalties (for identical aircraft, positioned to fly very close to the vortex). This reduction in speed would require the formation to fly about 1,000 ft lower than the normal cruise altitude, or fly at a $5 \%$ higher lift coefficient. This latter option has the additional benefit that the total drag of the formation decreases for modest increases in lift coefficient. Slowing down is an intriguing option in that a reduction in cruise Mach number of even a few percent from today's speeds has the potential for significant reductions in environmental impacts, ${ }^{26}$ as well as synergistic savings with other next generation technologies such as natural laminar flow wings and open rotor engines. The disadvantage to slowing down is the increased flight time (about 10 minutes on an 8 hour flight) and associated higher costs.

Formations that fly with a larger lateral separation between aircraft allow for a slightly higher in-formation cruise Mach number (about 1.5\% below the nominal out-of-formation cruise speed with $5 \%$ span spacing). The tradeoff for the increased offset distance is a $5 \%$ increase in induced drag. There seems to be little incentive to flying any further from the vortex as the reduction in compressibility effects becomes disproportionately smaller, while induced drag savings decrease significantly.

The results in this paper provide some insight into the tradeoffs and limitations of flying at transonic speed in extended formations, but further exploration is needed. Subsequent studies to this initial work have examined the additional inviscid drag penalty of trimming the aircraft in formation, ${ }^{22}$ and the fuel and cost benefits of utilizing formation flight in optimized airline flight schedules. ${ }^{24}$ Future work could focus on incorporating more detailed geometries, using viscous solvers, investigating formations with more than two aircraft and with heterogeneous aircraft, designing multi-point optimal wings, and conducting additional flight tests.

\section{Acknowledgments}

The first two authors were part of a broader research program on formation flight at Stanford University that was kindly supported by Airbus UK. Researchers at NASA Ames were supported through the Aeronautics Mission Directorate's Fundamental Aerodynamics program and through contract NNA10DF26C.

\section{References}

${ }^{1}$ Boeing Commercial Airplanes, Current Market Outlook 2010-2029, 2010.

${ }^{2}$ Airbus, Global Market Forecast 2009-2028, 2009.

${ }^{3}$ Wieselsberger, C., "Beitrag zur Erklärung des Winkelfluges einiger Zugvögel," Z. Flugtechnik 63 Motorluftschiffahrt, Vol. 5, 1914, pp. 225-229.

${ }^{4}$ Lissaman, P. B. S. and Shollenberger, C. A., "Formation Flight of Birds," Science, Vol. 168, No. 3934, 1970, pp. 1003, doi:10.1126/science.168.3934.1003.

${ }^{5}$ Hummel, D., "Aerodynamic Aspects of Formation Flight in Birds," Journal of Theoretical Biology, Vol. 104, No. 3, 1983, pp. 321-347.

${ }^{6}$ Blake, W. and Multhopp, D., "Design, Performance and Modeling Considerations for Close Formation Flight," AIAA Atmospheric Flight Mechanics Conference and Exhibit, AIAA-1998-4343, August 1998, pp. 476-486.

${ }^{7}$ Wagner, E., Jacque, D., Blake, W., and Pachter, M., "Flight Test Results of Close Formation Flight for Fuel Savings," AIAA Atmospheric Flight Mechanics Conference and Exhibit, August 2002.

${ }^{8}$ Ray, R. J., Cobleigh, B. R., Vachon, M. J., and John, C. S., "Flight Test Techniques Used to Evaluate Performance Benefits During Formation Flight," TP-2002-210730, NASA, August 2002.

${ }^{9}$ Ning, S. A., Flanzer, T., and Kroo, I., "Aerodynamic Performance of Extended Formation Flight," Journal of Aircraft, Vol. 48, No. 3, May 2011, pp. 855-865, doi:10.2514/1.54636.

${ }^{10}$ Aftosmis, M. and Berger, M., "Multilevel Error Estimation and Adaptive h-Refinement for Cartesian Meshes with Embedded Boundaries," AIAA Aerospace Sciences Meeting, AIAA 2002-0863, January 2002.

${ }^{11}$ Nemec, M. and Aftosmis, M. J., "Adjoint Error Estimation and Adaptive Refinement for Embedded-Boundary Cartesian Meshes," AIAA Computational Fluid Dynamics Conference, AIAA 2007-4187, June 2007.

${ }^{12}$ Nemec, M. and Aftosmis, M. J., "Adjoint Sensitivity Computations for an Embedded-Boundary Cartesian Mesh Method," Journal of Computational Physics, Vol. 227, No. 4, 2008, pp. 2724-2742, doi:10.1016/j.jcp.2007.11.018. 
${ }^{13}$ Ning, A. and Kroo, I., "Compressibility Effects of Extended Formation Flight," 29th AIAA Applied Aerodynamics Conference, Honolulu, Hawaii, June 2011.

${ }^{14}$ Gerz, T. and Holzäpfel, F., "Wing-Tip Vortices, Turbulence, and the Distribution of Emissions," AIAA Journal, Vol. 37, No. 10, October 1999, pp. 1270-1276, doi:10.2514/2.595.

${ }^{15}$ Betz, A., "Behavior of Vortex Systems," TM-713, NACA, 1933.

${ }^{16}$ Delisi, D., Greene, G., Robins, R., Vicroy, D., and Wang, F., "Aircraft Wake Vortex Core Size Measurements," AIAA Applied Aerodynamics Conference, AIAA-2003-3811, June 2003.

${ }^{17}$ Holzäpfel, F., "Probabilistic Two-Phase Wake Vortex Decay and Transport Model," Journal of Aircraft, Vol. 40, No. 2, March 2003, pp. 323-331, doi:10.2514/2.3096.

${ }^{18}$ Wintzer, M., "Span Efficiency Prediction Using Adjoint-Drive Mesh Refinement," Journal of Aircraft, Vol. 47, No. 4, July 2010, pp. 1468-1470, doi:10.2514/1.C031049.

${ }^{19}$ Redeker, G., "A Selection of Experimental Test Cases for the Validation of CFD Codes," AGARD AR, Vol. 2, No. 303, August 1994.

${ }^{20}$ Aftosmis, M. J., Berger, M. J., and Alonso, J. J., "Applications of a Cartesian Mesh Boundary-Layer Approach for Complex Configurations," AIAA Aerospace Sciences Meeting, AIAA-2006-0652, January 2006.

${ }^{21}$ Vassberg, J. C., DeHaan, M. A., Rivers, S. M., and Wahls, R. A., "Development of a Common Research Model for Applied CFD Validation Studies," AIAA Applied Aerodynamics Conference, AIAA 2008-6919, August 2008.

${ }^{22}$ Kless, J., Aftomis, M., Ning, A., and Nemec, M., "Inviscid Analysis of Extended Formation Flight," AIAA Journal, (in press).

${ }^{23}$ Leoviriyakit, K. and Jameson, A., "Multi-point Wing Planform Optimization via Control Theory," AIAA Aerospace Sciences Meeting, AIAA 2005-0450, January 2005.

${ }^{24}$ Xu, J., Ning, A., and Bower, G., "Aircraft Route Optimization for Formation Flight," Journal of Aircraft, (in review).

${ }^{25}$ Shevell, R., Fundamentals of Flight, Prentice Hall, 2nd ed., 1989.

${ }^{26}$ Dallara, E. S. and Kroo, I. M., "Aircraft Design for Reduced Climate Impact," AIAA Aerospace Sciences Meeting, AIAA 2011-265, January 2011. 